cours d'eau, il fait connaître immédiatement son aviş motivé au ministre des travaux publics ainsi qu'au ministre de l'agriculture s'il s'agit d'un cours d'eau ne faisant pas partie du domainé public. Le ministre des travaux publics décide, d'accord aver le ministre de l'agriculture, pour les cours d'eau ne faisant pas partie du domaine public, s'il y a licu ou non de poursuivre l'instruction.

En cas de désaccord avec l'administration locale des domaines au sujet de la fixation de la redevance applicable aux usines projetées sur les conrs d'eau domanianx, l'ingénieur en chef en réfère immédiatement au ministre des travaux publics, qui statuc d'accord avec le ministre des finances.

$\Lambda$ rt. I6. - Si les ouvrages sont compris dana le ravon myriamétrique d'une enceinte fortifiée èt si les modifications an régime des eaux sont de nature à exercer une influcnce sur les inondations défensives, il est procédé à l'instruction suivant les règles édictées par les lois et décrets sur les travaux mixtes.

Art. 17. - L'ingénicur en chef à qui est confiée l'instruction envoie au préfet chargé d'en centraliser les résultats l'ensemble du dossier avec ses pronositions.

Art. I8. - Dès réception du dossier, le préfet statue sur la demande. S'il accorde l'antorisation, il envoic une copie de l'arrêté au ministre des travaux publics ainsi qu'au ministre de l'agriculture. s'il s'agit d'un cour's d'cau ne faisant pas partic du domaine public.

Fn cas de rejet de la demande, le préfet notifie immédiatement sa décision mativée au pétitionnaire.

Si les travaux s'étendent sur plusieurs dénartements, il est sta tué par un arrêté unique signé par lẹ préfets intéressés ; en cas de désaccord, les préfets statuent conformément aux instructions qui lui sont données par le ministre des travaux publics si les travaux sont projetés sur un cours d'eau domanial et par le ministre de l'agricullure dans les autres cas.

Les autorisations d'une durée supérieure à cinn ans sur les canaux de navigation ou les rivières canalisées sont accordées par décret sur rapport du ministre des travaux publics, après avis du comité consultatif des forces hydrauliques

Art. in - L'arcomplissement des formalités prévues au présent décret, ne devra pas dénasser une duréc de six mois Toutefois, cette durée sera angơmentée dı délai supplémentaire prévu à l'article 8

Art. 20. - Lorsqu'il y a lieu de prononcer le retrait ou la modification de l'autorisation par apnlication des articles i/4 et 45 de la loi du 8 avril $\times 898$, il est statué dans les formes ćtablies par les articles 9, то, тा, г2, т3, т4, г5, т6, г7 et 18 du présent décret.

L'enquête s'ouvre, en ce cas, sur les propositions formulées par les ingénieurs.

\section{TTTRF II}

RÉCOLEMENT DES TRAYAITY

Art. 2I' - A l'cxpiration du délai fixé par l'acte d'autorisation pour l'exécution des travaux, l'ingénieur ordinaire se transporte sur les lieux pour vérifier si les travaux ont été exécutés conformément aux dispositions prescrites et rédige un procès-verbal de récolement en présence du pétitionnairc, des maires ou de leurs représentants et des intéressés convoqués à ret effet dans les formes établies par l'article 6 du présent décret.

S'il résulte du scécolement que les travaux exécutés sont conformes aux conditions de l'autorisation, le préfet en prononce la réception.

Si les travaux sont de nature à causer des dommages, lo préfet met immédiatement le pétitionnaire en demeure de satisfaire, dans un délai déterminé, aux conditions de l'autorisation

A l'expiration de ce délai, si la mise en demeurc est restéc sana cffet, le préfet prend les mesures nécessaires pour faire cesser le dommage et prononce, s'il $y$ a lieu, le retrait de l'autorisation, dont il avise le ministre des travaux publics, ainsi que le ministre de l'agriculture, s'il s'agit d'un cours d'ean ne faisant pas partic du domaine publir.

\section{TITRE III}

\section{Dispositrons DIVERSES}

Art. 22. - Dans le délai d'un an à partir de la publication dui préscut décret, les propriétaiıcs des usines existantes non fondées en litre, et qui n’ont pas été régulièrement autorisées, secont tenus de demander l'autorisation prévue par la loi du I6 octobre ryin. La demande doit être accompagnée des renseignements prévus à l'article 3 ci-dessus et d'une copie des titres en vertu desquels l'étahlissement existe.

Au cas où la demande ne serait pas présentée dans le délai imparti, le préfet prut ooit procéder à la réglementation d'office, soit imposer au propriétaire, après avis du comité consultatif des forces hỵdrauliques, le rétablissement du libre écoulement des eaux.

Art. 23. - Lo ministre des travanx publics ef le ministre de lagriculture sont chargés, chacun on ec qui le concerne, de l'exécution du présent décret, qui sera publié au Journal Officiel de la Rémublique française et inșéré au Bulletin des Lois.

Fait à Rambouillet, le 3o juillet rq2o.

(A suivre)

P. Deschanel.

\title{
NOTE DE LA'RÉDACTION
}

Nous sommes heureux d'annoncer it nos lecteurs, que notre distingué collaborateur, M. Paul Botanult, Avocat à la Cour, donnera prochainement dans la Houille Blanche, une série d'études sur les points les plus intéressants de la mise en ceuve pratique de la loi des chules d'eau, Cahier des charges, restitution de l'énergie supprimée, etc., etc.

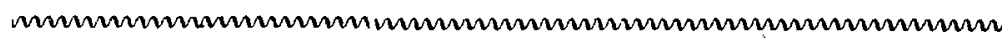

SUR UNE MÉTHODE THERMOMÉTRIQUE

DE

\section{MESURE DU RENDEMENT DES}

\section{TURBINES HYDRAULIQUES}

\section{I. - Considérations générales.}

Dans len opérations de délormination du rendement des machines, à quelque lype qu'elles appartiennenl du reste, on s'est eflorcé depuis longlemps de mesurer directement, par voie calorimétrique ou thermique, la fraction de la puissance disparaissanl dans len dites machines sous forme de pertes. Il est bien évident, dans l'élal actuel des théories scientifiques, que la différence entre la puissance fournie $P$ et la puissance utile $\mathrm{P}$, représentant la puissance dégradée et inutilisable, se retronverail sons forme de chaleur dans une enceinte supposće adiathermane, qui envelopperait théoriquement la machine de toutes parts, le fluide moteur évoluant Jui-même à l'inlérjeur de cette enceinte. Une circulation d'eau, convenablement réglće dans l'enceinte, dónnerajt par la différence des températures amont ct aval du liquide, en y joignant la connaissance du débit de l'eau calorimétrique, la puissance dissipée dans la dite machine.

On concoit les difficultés expérimentales soulevées par l'application de cette mélhode. Nous ne nous y arrêterons pas. Notamment, dans, Jes machines électriques, ce n'est qu'une petite fráction des pertes qui devient accessible directement. sous forme thermiruır immédiate, à savoir : les pertes dans les enroulements. Certaines installations de Gentrales prévoient en particulier, pour raisons de sécurité, la mise en évidence permanente de res échauffements, mais ce ne sont là que des cas isolés 
Dans le cas des turbines hydrauliques, une telle méthode de mesure du rendement global, par voie thermométrique ou calorimétrique est-elle possible ? Qu'on ne vienne pas dire a priori que le principe de la méthode est bon ou mauvais ! L'expérience seule jouera le rôle de critère. Il nous a semblé logique personnellement de nous affranchir provisoirement de tout esprit de critique préalable et de rapprocher purement et simplement les chiffres déduits de l'application de cette méthode de ceux apportés par d'autres méthodes. Ce sont les faits seuls qui parleront, c'est aux faits seuls que nous demanderons la justification ou l'infirmation de notre esquisse de théorie, ne désirant qu'une chose, ouvrir une discussion...

\section{II. - Exposé du principe de la Méthode Thermométrique.}

La méthode susvisée est donc basée sur lá remarque que, dans une turbine hydraulique, les quantités de chalcur correspondant aux pertes d'énergie sont employécs à échauffer l'eau qui, en même temps que celui de fluide moteur, sc trouve jouer le rôle de réfrigérant.

Soit une turbine hydraulique absorbant, en régime établi, un débit de Q litres par seconde, sous une hauteur de chûte, lue au manomètre, de $\mathrm{H}$ mètres.

La puissance-eau fournie à la turbine est : (I)

$$
\text { 'P }=\text { Q.H. Kgm/sec. }
$$

Si nous admettons, par hypothèse, que les diverses pertes d'énergie dans cette turbine se transforment intégralement en chaleur, évacuée par l'eau elle-même (nous verrons plus loin que ceci semble à peu près réalisé), l'échauffement de l'eau (différence entre la température $\Theta_{1}$ à l'entrée et celle $\Theta_{2}$ à la sortie de la turbine, en degrés centigrades) sera proportionnel aux pertes $p$, et l'on pourra écrire :

$$
\text { pertes } p=425 \mathrm{Q}\left(\Theta_{2}-\Theta_{1}\right) \mathrm{kgm} / \mathrm{sec} \text {. }
$$

Le rendement de la turbine sera alors :

$$
\begin{aligned}
& \eta=\frac{\mathrm{P}-\mathrm{P}}{\mathrm{P}}=\frac{\mathrm{QH}-425 \mathrm{Q}\left(\Theta_{2}-\Theta_{1}\right)}{\mathrm{Q} \cdot \mathrm{H}}=\frac{\mathrm{H}-425\left(\Theta_{2}-\Theta_{1}\right)}{\mathrm{H}} \\
& \text { (2) } \quad \eta=1-\frac{425\left(\Theta_{2}-\Theta_{1}\right)}{\mathrm{H}}
\end{aligned}
$$

expression dans laquelle le débit $Q$, qui était précisément le facteur difficilement mesurable, est éliminé.

La valeur du rendement serait ainsi obtenue instantané. ment par une lecture de différence de température et une lecture de pression manométrique.

Evaluons l'ordre de grandeur de cet échauffement. :-i lon suppose avoir affaire à une chûte de 3 oo mètres, et une turbino ayant environ 0,80 de rendement, l'échauffement de l'eau sera :

$$
\Theta_{2}-\Theta_{1}=\frac{p}{425 \mathrm{Q}}=\frac{0,20 \mathrm{Q} \cdot \mathrm{H}}{425 \mathrm{Q}}=\frac{0,2 \mathrm{H}}{425 \mathrm{Q}}=0^{\circ}, 141
$$

Cet échauffement est donc proportionnel à la hautıur de chûte ; pour une chûte de 3o mètres, il serait de $0^{\circ}$ or $4 \mathrm{I}$.

Il faut donc, pour exécuter des mesures suffisanmont précises, disposer de thermomètres, simples ou différenlicls, capables de mesurer au $1 / 100^{\circ}$ près la différence $\Theta_{2}-\AA_{1}$

La réalisation de cette condition est facile pour des chûtes supérieures à roo mètres, et elle n'est pas impossible juur des chûtes de hauteurs plus faibles.

Des thermomètres a mercure conviennent fort bien pour cès mesures ; également, avec certaines précautions, des thermomètres à gaz.

L'emplor de couples thermo-électriques " fer-constantan " permettra aussi la mesure avec précision, et elle scra instantanée, puisque différentielle (fig. I).

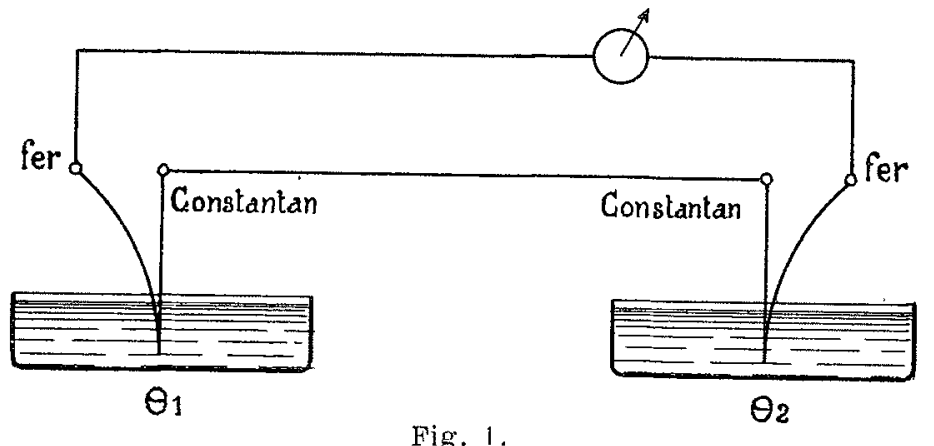

Les deux couples sont respectivement plongés dans les enceintes $A$ et $B$ (conduite forcée et canal de fuite). Ils sont connectés en opposition sur le galvanomètre $G$ ct donnent lieu à une dévialion proportionnelle à $\Theta_{2}-\Theta_{1}$. Si l'on intervertit les couples, on doit retrouver la même déviation, mais de sens inverse. Le maximum de sensibilité sera obtenu avec des fils de connexion de résistance négligeable, et un galvanomètre $G$ de résistance égale à celle de la somme des deux couples. On peut, en outre, multiplier l'effet au moyen de plusieurs couples associés en série ou en parallèle.

Le galvanomètre sera étalonné empiriquement, mais avec toute la précision nécessaire.

\section{III. - Analyse des pertes dont les turbines sont le siège.}

Il reste à tenter de justifier l'hypothèse, faite au début, que les pertes d'énergie dans la turbine se traduisent en chaleur pratiquement toute évacuée par l'eau motrice.

Nous le répétons : nous n'émetions ici aucune affirmation a priori. Nous ne donnerons au contraire cette esquisse de théorie que pour couvrir éventuellement des résultats numériquement concordants et acceptables, si l'expérience démontre qu'il en est ainsi.

Les pertes d'énergie sont en effet les suivantes :

$\mathrm{I}^{\circ}$ Pertes par brassage et frottement de l'eau, sur les aubes, fuites, remous et tourbillons. - Elles échauffent l'eau intimement, d'une part, et superficiellement la roue de la turbine et les parois, d'autre part, mais en raison du contact intime de ces parties avec l'eau, on peut admettre que l'eau emporte ces calories, ainsi que celles dúes à la ventilation par la roue de turbine.

Etant donnée la faible élévation de température de l'eau, il ne semble pas y avoir à craindre que la masse de la turbine joue le rôle de radiateur, dans l'ambiance, d'une portion appréciable de la chaleur dégagée, ni même inversement que l'ambiance elle-même, à températuré généralement plus élevée que celle de l'eau, réchauffe celle-ci, par conduction à travers la turbine, d'une quantité anoréciable, d'autant moins que l'eau circule avec une grande vitesse linéaire.

$2^{\circ}$ Pertes par pulvérisation et vaporisation de l'eau. Comme il ne s'échappe, dans le canal de fuite, que de l'eau et pas de vapeur d'eau parce que toutes les poussières ct vaneurs (en admettant qu'il s'en produise par chocs) sé résolvent finalement au, sein du liquide, en Iui restituant, leur chaleur aussitôt, il est raisonnable d'admettre que c'est encore l'eau aui emporte intégralement cette quantité do chaleur éventuelle. 
$3^{\circ}$ Pertes par frottement dans les paliers de la turbine. - C'est la seule partie des pertes dont la chaleur ne soit certainement pas emportée dans son intégrité par l'eau. Mais encore, comme cette partie des pertes n'est qu'une faible fraction des pertes totales et que, en outre, une grande partie de la chaleur correspondante est transmise à l'eau par l'arbre de la turbine, on pourrait se croire autorisé à négliger cette cause d'erreur. En effet, le rayonnement des paliers dans l'ambiance est, en réalité, très faible et l'on pourrait d'ailleurs aménager autour de ces paliers une circulation d'eau prise dans la bâche de la turbine el rejetée dans le canal de fuite.

En résumé, pour ces divers motifs, puis en raison de la rapidité de circulation de l'eau et de sa faible différence de tompérature avec l'ambiance immédiate, terre ou air, on peut se croire autorisé à admettre que l'eau évacue en chaleur la totalité de la puissance correspondant aux diverses pertes.

Cette méthode doit donc pouvoir donner des résultats cxacts, à I \% ou $2 \%$ près.

\section{IV. - Mode opératoire. - Précautions à prendre dans la mesure des températures.}

$x^{\circ}$. Dans le canal de fuite, placer le thermomètre dans un cndroit où l'eau est aussi calme que possible et au milieu du canal pour éviter l'effet des parois.

$2^{\circ}$ Dans la conduite forcée, placer le thermomètre en un point où la vitesse de l'eau est faible et ne puisse produire d'erreurs par frottement de l'eau sur le thermomètre.

Si c'était nécessaire, on prendrait la température $\Theta_{1}$ dans la chambre de mise en charge de la conduite forcée, et l'on mesurerait ainsi le rendement global conduite-turbine, d'où l'on déduirait celui de la turbine, le rendement de la conduite forcée étant égal au rapport entre les pressions manomélriques en charge et à l'arrêt, près de la turbine.

$3^{\circ}$ Prendre garde à ce fait que la température propre de l'eau peút varjer en certaines saisons de $\mathrm{r}^{\circ}$ à $3^{\circ}$ par jour. soit $0^{\circ}, 04$ à $0^{\circ}, \mathrm{I} 2$ par heure et même, à certaines heures de la journée, de $0^{\circ} 5$ à $I^{\circ}$ par heure. Il faut done que les mesures de $\Theta_{1}$ et $\Theta_{2}$ soient aussi simultanées que possible.

\section{REMARQUES}

Remaroue I. - Dans l'hypothèse de l'exactitude de la méthode, les appareils de mesure des températures pourraient rester en permanence installés et donner à chaque instant, et à toutes les charges des turbines, la valeur de leur rendement.

Installé pour chaque turbine, ce système donnerait an chef d'usine d'utiles indications sur l'éfat des organes raubes of distributeurs).

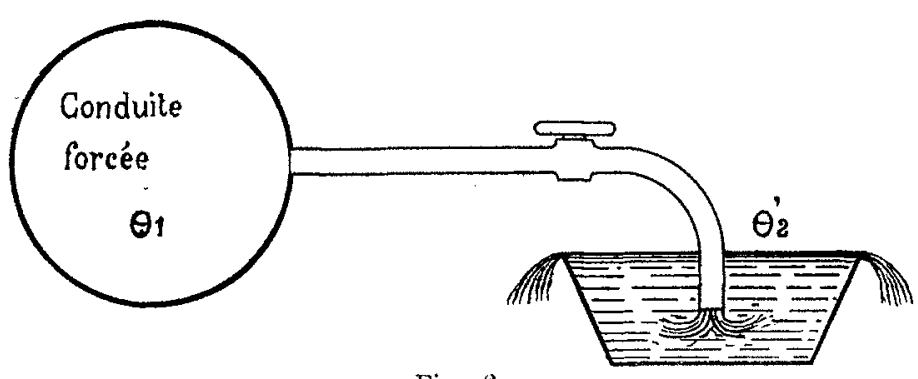

Fig. 2.

Remarque II. - Si l'on soutire de l'ean de la conduité directement dans une enceinte, sans lui faire produire de travail mécanique, son énergie intrinsèque se transforme intégralement en chaleur (rendement $=0$ ), d'où : $\theta_{2}^{\prime}=$ température de l'cau calme soutirée).

$$
\eta^{\prime}=1-\frac{425\left(\Theta_{2}^{\prime}-\Theta_{1}\right)}{H}=0
$$

d'où :

$$
\Theta_{2}-\Theta_{1}=\frac{H}{425}
$$

pour

$$
\mathrm{H}=300 \mathrm{~m}, \quad \Theta_{2}^{\prime}-\Theta_{1}=00,707
$$

On peut, au moyen de cette remarque, soit contrôler lés autres mesures, soil connaissant $\Theta_{2}^{\prime}$ en déduire $\Theta_{1}$ pour évaluer ensuite $\Theta_{2}-\Theta_{1}$ de la turbine, si l'on ne peut mesurer $\Theta_{1}$.

\section{Soit $\mathrm{H}_{m}$ la pression manométrique en charge.}

Par conséquent, connaissant $\Theta^{\prime}{ }_{2}$ et $\Theta_{2}$, lc rendement de la turbine s'exprime par :

$$
r_{1}=\frac{425\left(\Theta_{2}^{\prime}-\Theta_{2}\right)}{\mathbf{H}_{m}}
$$

Ce procédé peut, dans certains cas, être plus commode, évitant les difficultés de prise' de température dans la conduite sous pression.

Corrections à apporter aux formules précédentes. - Ces formules sont relatives à de l'eau pure. Si, commc il arrive parfois, l'eau est plus ou moins chargéc de sable (au point que sa densité peut être accrue de $5 \%$ ou même davantage), les mesures, pour être précises, demandent une correction de ce fait.

Si ó est la densité de l'eau sablonneuse, et $c$ sa capacité thermique, la formule du rendement devient :

( w) $\eta=1-\frac{425 . c\left(\Theta_{2}-\Theta_{1}\right)}{\mathrm{H}}=1-\frac{425 . c . \delta\left(\Theta_{2}-\Theta_{1}\right)}{\mathrm{H}_{m} \text { lue au manomètre. }}$

H étant la hauteur de chn̂te réelle $=\frac{\text { pression manómétrique II }}{\delta}$ agissant sur la turbine.

Les facteurs de correction c el o sont aisés à fixer dans chaque cas. Cette correction est d'ailleurs peu sensible, en général ; si le poids du litre d'eau reste assez voisin de I $\mathrm{kg}$., on n'aura pas à en tenir compte.

On a, en effet, dans ce cas (débit de $Q$ litres par seconde) :

Pression manométrique $\mathrm{H}^{\prime}=\delta \mathrm{H}$ (on lit $\mathrm{H}^{\prime}$ mètres au manomètre) ;

Puissance-eau totale : $\mathrm{P}=\mathrm{QH}^{\prime}=\mathrm{Q} \delta \mathrm{H} \mathrm{kgm} / \mathrm{sec}$;

Pertes totales : $p=425, c .8 . Q\left(\Theta_{2}-\Theta_{1}\right) \mathrm{kgm} / \mathrm{sec}$. (car $c$, chaleur spécifquue, est la quantité de chaleur par $\mathrm{kg}$. de liquide et par degré centigrade).

$$
\text { rendement } \begin{aligned}
\eta & =\frac{\mathrm{P}-P}{\mathrm{P}}=\frac{\mathrm{Q} \delta \mathrm{H}-425 \cdot c \cdot \mathrm{Q}\left(\Theta_{2}-\Theta_{1}\right) \cdot \delta}{\mathrm{Q} \cdot \delta \cdot \mathrm{H}} \\
& =1-\frac{425 \cdot c \cdot\left(\Theta_{2}-\Theta_{1}\right) \delta}{\delta \mathrm{H}} \\
& r_{i}=1-\frac{425 \cdot c \cdot \delta\left(\Theta_{2}-\Theta_{1}\right)}{\mathrm{H}_{m}^{\prime} \text { lue au manomètre. }}
\end{aligned}
$$

ou encore, si l'on connaît $\Theta_{2}^{\prime}$ Tdéfinie comme précédemment), soit la température de détente dans "un récipient sans travail mécanique] el $\Theta_{2}$ au canal de fuite :

$$
\eta=\frac{425 \cdot c \cdot s\left(\Theta_{2}^{\prime}-\Theta_{2}\right)}{H_{m}^{\prime}} \text { Jue au manomètre. }
$$




\section{V. - Quelques essais d'application de la méthode thermométrique. - Chiffres et observations.}

A l'aide de deux thermomètres à mercure sensibles, gradués de $0^{\circ}$ à 25 , et donnant une dilatation de $40 \mathrm{~mm}$. environ par degré centigrade, gradués en $1 / 20$ de degré, on a pu faire des mesures précises déjà sur trois chûtes différentes, en Savoic, dans les úsines suivantes que nous dénommerons respectivement $\mathrm{A} B$ et $\mathrm{G}$.

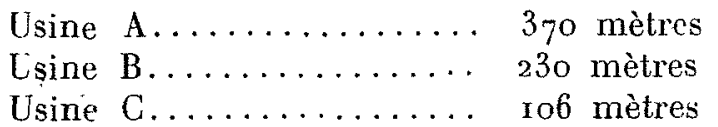

Ci-après les résultats numériques obtenus :

Dans chaque cas, on a procédé comme suit :

$I^{\circ}$ Mesure de $\Theta_{2}$ dans le canal de fuite, avec les deux thermomètres ensemble, pour contrôle, en notant l'instant :

$2^{\circ}$ Mesure de $\Theta_{2}^{\prime}$ (de l'eau détendue dans un récipient), si l'on peut s'exprimer ainsi) avec les deux thermomètres, en notant l'instant;

$3^{\circ}$ Nouvelle mesure de $\Theta_{2}$, en notant l'instant ;

$4^{\circ}$ Nouvelle mesure de $\Theta_{2}^{\prime}$, en notant l'instant

Ces quatre mesures effectuées à intervalles aussi rapprochés que possible. Mesure de la-pression manométrique correspondante.

(Il s'agissait là de premières mesures, et l'on peut opérer de façon plus rationnelle, comme il sera indiqué par la suite).

On a relevé les chiffres suivants :

\section{Usine A}

Quatre unités en marche, dont trois de 2.200 HP et un de 4.80o HP ; l'une des turbines de 2.200 HP est du type Girard, assez. usagée; les trois autres turbines sont du type Pelton, modernes et neuves. Ces quatre unités sont à peu près à la charge $3 / 4$.

La charge est assez constante ; les régulateurs des turbines sont lajssés en action. On mesurera donc le rendement global des quatre turbines à la charge considérée.

Il $y$ a, en outre, une turbine d'excitatrice Girard, de ro $\mathrm{HP}$, ̀̀ $3 / 4$ de charge.

\begin{tabular}{|c|c|c|c|c|c|}
\hline \multirow{2}{*}{ Temps } & \multicolumn{2}{|c|}{$\Theta_{2}$ (Caaal de fuite) } & \multicolumn{2}{|c|}{$\Theta_{2}^{\prime}$ (Eau dẻtendue) } & \multirow{2}{*}{$\begin{array}{c}\text { Manomètre } \\
\text { aux } \\
\text { turbines }\end{array}$} \\
\hline & Therm.no 1 & Therm.no2 & Therm. no 1 & Therm.no2 & \\
\hline $\begin{array}{c}1 \mathrm{r} \theta \\
\text { mesure }\end{array}\left\{\begin{array}{l}16^{\mathrm{h}} 49^{\mathrm{m}} \\
16^{\mathrm{b}} 51^{\mathrm{m}}\end{array}\right.$ & $\begin{array}{c}15^{\circ}, 59 \\
\ldots \ldots\end{array}$ & $\begin{array}{l}15^{\circ}, 63 \\
\ldots \ldots\end{array}$ & 160,27 & $16^{\circ}, 305$ & $\begin{array}{l}360 \mathrm{~m} \\
360 \mathrm{~m}\end{array}$ \\
\hline $\begin{array}{c}2 \mathrm{me} \\
\text { mesure }\end{array}\left\{\begin{array}{c}16^{\mathrm{h}}, 53 \mathrm{~m} \\
16^{\mathrm{b}} 55^{\mathrm{m}}\end{array}\right.$ & $15^{a}, 625$ & $\begin{array}{c}150.665 \\
\ldots .\end{array}$ & 16,30 & $16^{\circ}, 34$ & $\begin{array}{l}36 r \mathrm{~m} \\
360^{\mathrm{m}}\end{array}$ \\
\hline
\end{tabular}

On voit d'après ces chiffres que, dans l'intervalle de quatre minutes, la température de l'eau a subi une variation propre de $0^{\circ}, 035$, soit donc $0^{\circ}, 017$ par deux minutes (ou $0^{\circ}, 52$ par heure), en augmentation à cette heure de la journée.

Les valeurs de $\Theta_{2}^{\prime}$ seront donc à diminuer respectivement, de cette quantité $0^{\circ} \mathrm{O} 7$, pour les ramener aux instants respectifs où ont été pris les $\Theta_{2}$.

On trouve ainsi, d'une façon très concordante :

$$
\Theta_{2}^{\prime}-\Theta_{2}=0^{\circ}, 66
$$

D'où le rendement global des turbines :

d'où :

$$
r=\frac{425\left(\Theta_{2}^{\prime}-\Theta_{2}\right)}{\mathrm{H}}=\frac{425 \times 0,66}{360}
$$

$$
\eta=0,78
$$

ce qui constitue un chiffre très probablement exact et en tous cas entièrement acceptable dans les conditions susénoncées.

\section{USINE B}

Deux unités en marche, à $2 / 4$ do chargc environ, dont I turbine de r.400 HP-Pclton moderne usagée

I turbine de 600 HP-Girard, ancienne usagée.

\begin{tabular}{|c|c|c|c|c|c|}
\hline \multirow{2}{*}{ Temps } & \multicolumn{2}{|c|}{ (Canal de fuite) } & \multicolumn{2}{|c|}{ (Eau détendue) } & \multirow{2}{*}{ Manomètre } \\
\hline & Therm.no 1 & Therm.no2 & Iherm.no1 & Therm no 2 & \\
\hline $\begin{array}{c}\text { ire }^{\mathrm{mes}} \\
\text { mesure }\end{array}\left\{\begin{array}{c}18^{\mathrm{h}} 2^{\mathrm{m}} \\
18^{\mathrm{h}} 6^{\mathrm{m}}\end{array}\right.$ & $\cdots \ldots \ldots$ & $\begin{array}{c}\ldots \ldots \\
16^{0}, 082\end{array}$ & $16,0,435$ & 160,475 & $\begin{array}{l}224^{\mathrm{m}} \\
224^{\mathrm{m}}\end{array}$ \\
\hline $18^{1 \mathrm{~h}} 11^{\mathrm{m}}$ & . & . . . & 160,383 & $16^{\circ}, 420$ & $22 / \mathrm{m}$ \\
\hline
\end{tabular}

Plus I excitatrice (turbine Pelton) de 500 HP.

Et I excitatrice (turbine Girard) de 30 HP.

Variation propre de température de l'eau (refroidissement) de $0^{\circ}, 05$ en neuf minutes, soit $0^{\circ}, 022$ en quatre minutes.

Les valeurs de $\Theta_{2}$ sont donc ici à majorer de cette quan-. tité.

On obtient alors :

$$
\Theta_{2}^{\prime}-\Theta_{2}=0^{\circ} 37
$$

D'où le rendement global des turbines :

$$
n_{1}=\frac{425 \dot{x} 0^{\circ}, 37}{224}=0^{\circ}, 705
$$

chiffre assez vraisemblable dans cette circonstance.

\section{UsINE C}

\begin{tabular}{|c|c|c|c|c|c|}
\hline \multirow{2}{*}{ Temps } & \multicolumn{2}{|c|}{ (Candl de fuite) } & \multicolumn{2}{|c|}{ (Eau détendue) } & \multirow{2}{*}{ Manomètre } \\
\hline & Therm.nol & Therm.no 2 & Therm. $\|^{\circ} 1$ & Therm.no 2 & \\
\hline mesure $\left\{\begin{array}{l}10^{\mathrm{h}} 3 \mathrm{~m} \\
10 \mathrm{~h} 7 \mathrm{~m}\end{array}\right.$ & $\begin{array}{l}10 \cdot, 12.5 \\
\ldots \ldots \ldots\end{array}$ & $\begin{array}{r}100,075 \\
\ldots \ldots \ldots\end{array}$ & $10^{\circ} 285$ & 100,24 & $\begin{array}{l}104^{\mathrm{m}} \\
104^{\mathrm{m}}\end{array}$ \\
\hline $2^{\text {me }}\left\{\begin{array}{l}10 \mathrm{~b} 9 \mathrm{~m} 30 \mathrm{~s} \\
10 \mathrm{~h} 11 \mathrm{~m}\end{array}\right.$ & $\begin{array}{l}100,15 \\
\ldots \ldots\end{array}$ & $\begin{array}{c}100,10 \\
\ldots \ldots\end{array}$ & 100,35 & $10^{\circ}, 30$ & $\begin{array}{l}104^{\mathrm{m}} \\
104^{\mathrm{m}}\end{array}$ \\
\hline
\end{tabular}

Deıx unités en marche, à $\mathrm{x} / 4$ de charge environ, soit deux turbines de 200 HP, Escher-Wyss, état (?). Charge très variable (tramway); régulateur en action; débit faible : mince lame d'eau dans le canal de fuite.

Entre io h. 3 et to h. $9 \mathrm{~m}$. I/2, échauffement propre de $0^{\circ}, 025$ en $6 \mathrm{~m}$. 5 , donc pour la première mesure, échauffement propre de $o^{\circ}$,or 5 en $4 \mathrm{~m}$.

On en déduit, pour la première mesure :

D'où :

$$
\Theta_{2}^{\prime}-\Theta_{2}=0^{\circ}, \mathrm{r} 5
$$

$$
r_{1}=\frac{425 \times 0.15}{104}=0,61
$$


Entre ro h. 7 et Io h. II, échauffement propre de $0^{\circ}, 06$ en $4 \mathrm{~m}$., donc pour la prenière mesure, échauffement propre de 0,023 en $\mathrm{I} \mathrm{m}$. $\mathrm{i} / 2$.

On èn déduit, pour la deuxième mesure :

$$
\begin{array}{cc}
\text { D'où : } \quad & \Theta_{2}^{\prime}-\Theta_{2}=0^{\circ}, 177 \\
& \eta=\frac{425 \times 0^{\circ}, 177}{104 .}=0,72
\end{array}
$$

On se trouvait là dans de mauvaises conditions pour exécuter des mesures, surtout en raison de la charge très variable, qui a donné lieu visiblement à des erreurs de lecture. Néanmoins, on entrevoit la possibilité ici encore, c'està-dire même pour une chûte de roo mètres, d'appliquer fructueusement celte méthode rapide, avec l'espoir d'en tirer une suffisante précison.

\section{CONCLUSION}

Ces mesures sous trois chûtes différentes, opérées sur des ensembles de turbınes, ont fourni des chiffres très concordants avec ce que nous connaissions par ailleurs de ces machines, en dépit des conditions rudimentaires dans lesquelles nous avons opéré.

II semble très possible d'admettre qu'en observant un crrtain nombre de précautions nécessaires, dont i] est fait mention ci-après, on puisse arriver à une véritable précision, peul-être à r \% ou $2 \%$ près) par cette méthode.

Le degré de précision (si l'on admet que le manomètre soit exact à moins de $x \%$ près, ce qui est réalisable), sera lié, toujours sous réserve de la valeur théorique de la méthode, à l'exactitude de la mesure de $\Theta_{2}^{\prime}-\Theta_{2}$, ou de $\Theta_{2}-\Theta_{1}$.

Or, Ins causes d'erreurs possibles qui ont apparu sont principalement les suivantes :

$\mathrm{I}^{\circ}$ Variations diurnes de la température de l'eau.

$2^{\circ}$ Effet de la capacité thermique des' masses en jeu : lurbine, parois du canal de fuite, masse d'eau contenue entre l'échappement de la turbine et le point où l'on prend la température $\Theta_{2}$, cet effet pouvant se faire sentir sensiblement si le régime de marche est variable.

$3^{\circ}$. Effet de la capacité thermique du tuyau, robinet et récipient avec lesquels on " détend " de l'eau de la conduite forcée.

Précautions à prendre. - Pour éviter ces diverses causes d'erreurs ou tout au moins pour réduire leur importance xu minimum, on devra, dans la mesure du rendement d'une turbine :

D'abord, opérer à régime constant pour chaque degré de charge, en libérant-la turbine de son régulateur, et en maintenant la charge aussi constante que possible, pour que la vitesse ne varie pas trop.

Pour chacrue degré de charge, attendre que les régimes do temnérature se soient établis (3 on / minutes suffisent) on toutes les parties, turbine'el canal de fuite.

Ne pas prendre les températures dans los remous et tourbillons, mais en des endroits aménagés à cet effet. où l'ean circulante est calme et pas tron rapide, pour éviter l'effet de frottement sur le thermomètre.

Dans la mesure de $\Theta_{2}^{\prime}$, dans un récinient léger et assez netit, avoir un débit d'eau convenable nar rapport à sa contenance, nour aue l'énergie de l'eau se transforme en chaleur uniquement dans le récipient. sans projections an dehors (disposition de chicanes, etc...). que l'ean déborde abondamment, maịs sans vitesse, du récipient. An moment de la lecture, faite après un temps suffisant, pour quei l'équilibre de lempérature soit réalisé, fermer le robinet pour. que l'cau soit calme et lire immédiatement.

Tenir un comple exacl, par des calculs appropriés, de la variation diurne, ascendante ou descendante, de la température propre de l'eau, cette variation étant parfois très scnsible, comme on l'a vu ; emploi de mesures simultanées, au signal, si on le peut, à l'aide de thermomètres soigneusement comparés.

Si l'on mesure le rendement par la formule (2), (mesure de $\Theta_{2}-\Theta_{1}$ ), la mesure de $\Theta_{1}$ nécessite des thermomètres spéciaux, résistant à la pression; se méfier des effets de celle-ci sur les indications du thermomètre (compression des parois). Si l'on mesure $\Theta_{1}$ dans la chambre de mise en charge, à l'entrée dans la conduite, tenir compte du temps d'écoulement pour arriver à la turbine, car des mesures simultanées ne conviendraient plus alors.

Enfin, si l'on juge à propos de tenir comple des pertes dans lc ou les paliers de la furbine, on peul, soil on faire une évaluation approximative, soit évacuer, dans le canal de fuite, les calories cui proviennent de ces perles, par une faible circulation d'ean prélevée dans le canal de fuite luimême à l'aide d'une pompe

Cette correction doit être d'ailleurs de peu d'effet on général (1\% probablement, pour les raisons indiquées déjà).

En terminant, et au risque de nous faire laxer de monotonic, nous ne saurions trop insister à nouveau sur le caractèrc hypothétique d'exactitude philosophique de la méthode que nous renons d'exposer.

Nous espérons que les quelrnes chiffres el quelques résultats d'essais rapportés plus haut démontreront notre varfaite bonne foi en la matière Nolre sincère espérance est qu'un mouvement d'opinion sera ainsi créé autour d'une question d'une importance capilale. Nous accueillerons avec le plus grand plaisir, nous sollicitons même les explications, observations ou suggestions qui pourraient nous être présentées par nos confrères et amis de l'industric.
16 nồt 1920
I. Barmiltion et A. Poirson.

\section{L'ÉTUIDE DES COUPS DE BÉLIER}

DANS LES

CANALISATIONS MÉTALLIQUES SOUS PRESSION

\section{RÉSULTATS OBTENUS PENDANT LA GUERRE (sUiTE)}

6. Transmission du coup de bélier provenant d'une fermeture brusque. - L'un de nous a montré que les réflexions qui se produisent dans une conduite à caractéristiques variables aux jonctions des divers tronçons ont pour effet de modifier complètement le phénomène de- la transmission intégrale dont nous avons parlé plus haut dans lc cas des conduiles à caracléristique unique. La valeur du coup de bélier au moment où l'onde arrive en haul sera :

$$
\begin{gathered}
i=n-1 \\
y=y_{0}+f_{1} \Pi\left(1-\frac{\alpha_{1}+\alpha_{l+1}}{\alpha_{2}+\alpha_{i+1}+1}\right) \\
i=1 \\
\alpha \text { étant le rapport } \frac{a}{\mathrm{~S}}
\end{gathered}
$$

